since been proved experimentally on animals, as well as the fact of the passage of the ovum through the uterus into the opposite tube.

There are points of interest in the changes which the foctus undergoes in the abdominal cavity that may be in place to mention here. One of these is the socalled lithopadion or stony foctus. 'This is one that has been carried a long time and still preserves its external features, like a mummy, while all its tissues arc infiltrated with lime. The specimen from the Warren Museum here shown was carried twenty-nine years. The longest recorded case is somewhat over fifty years.

In other cases the fotus is quite quickly destroyed after death, and only the macerated bones are found. These are often voided spontaneously with the external opening of an abscess, as in the earliest recorded case, already mentioned.

How these two distinct processes could be brought about was shown by the experiments of Leopold, who introduced the embryos of one rabbit into the abdominal cavity of another; and he concludes that, in order for the fotus to be mummified, it must be enclosed in a sac of proper membranes, and excite very little inflammation. If this is not the case, the soft parts are quickly destroyed and a skeleton is the result.

There remains only to call attention to the changes which take place in the uterus itself. It is usually stated that it is somewhat enlarged and a decidua is formed within it. Some of these specimens which are shown from the museum agree with this, especially the cases of interstitial pregnancy. But others give little evidence in the uterus of the pregnancy which you see has taken place in the tube.

There remains only one other condition to mention, and that is where the foctus is found within the uterine walls. How it gets there is not as yet fully understood; but it is supposed to be in some way connected with a development of the ovum in Giirtner's canal, which has a connection with tho Fallopian tube.

In closing, it may be said that the cases of extraperitoneal pregnancy are those where the foctus has been first formed in the tube or abdomen and through rupture has found its way into the layers of the broad ligament or beneath the peritoneum.

\section{EXTRA-UTERINE PREGNANCY.}

BY RDWARU IRYNOLDS, M.D.

Ar the first meeting of the Committee appointed by this Society to report upon the present condition of medical opinion with regard to the subject of extrauterine pregnancy, it was decided that the subject should be sub-divided among ourselves in such a way that 1 should have the honor of presenting to you a review of the recent literature of the subject, and of the opinions of those who have made themselves best known in commection with the questions of its pathology, the classification of its varieties, the possibilities of an early diagnosis, and the therapentic responsibilities which that must entail.

To the great gratification of the Committee, Dr. Wm. F. Whitney has, however, been kind enough to agree to open this discussion by the exhibition of pathological specimens, and a review of the pathological points which his large experience has led him to think important.

I Iead before tho Boston Obstetrionl Suoioty, Novomber 12, 1882.
I feel that I must preface my own share of this work by reminding you that the subject has so recently come into prominence that many of the most important points are still under discussion; and by stating that in the midst of the uncertainties which still beset it, it is impossible to do anything more with many of the questions involved than to present them in a shape for discussion, to quote the dicta of different authorities, and finally to postulate briefly the few points which seem to have become permanently settled.

The still unsettled state of opinion with regard to the frequency of extra-uterine gestation makes its difficult to reach any satisfactory conclusion upon this point ; and the difference of opinion which obtains has been the cause of considerable and, unfortunately, somewhat acrimonious discussion. I believe that this alleged difference in frequency is due partly to a more extended interest in the early diagnosis of the affection in some localities than in others, and partly to a more active habit of reporting all cases in the first-named class of cities. There is probably no city in this coun. try, and perhaps none in the world, in which so many instances have been reported in proportion to the population, as in Philadelphia; few, if any, cities of the first cluss (in a medical sense) in which so few cases have been reported, as in Boston. But I believe that we shall find this evening that the difference between the number of cases which have been ohserved here, and those which have been seen in Philadelphia even, is no more than would be explained by the smaller size of our cily, when taken in connection with the fact that those men here who have had opportunities for observing such cases have not usually reported them, and that the interest of the general profession in the early diagnosis is therefore but partially awakened. If this explanation bo true, it is certainly to be hoped that this discussion to-night may result in the detection and cure of a large number of future cases, which otherwise would have passed on to incurable disease or death.

1)r. H. F. Formad, the coroner's physician of Philadelphia, had seen upon the post-mortem tablo in 1890 , more than thirty cases in which death was due to an undiagnosed ectopic pregnancy, all collected within the three or four preceding years; and there may have been, and no doubt were, in previous years, equally $\operatorname{large}$ numbers which fuiled of recognition in Philadelphis.

If the gynecologists of this vicinity are ready to admit that they, in common with other practitioners, must in the past have seen many cases of unrecognized tubal pregnancy, is it not likely that the pathologists must be prepared to make the samo admission? I think we shall find our operators are prepared to state that they are constantly seoing a larger proportion of such cases, and 1 hopo that our pathologists will be able to make the same report.

But the undiagnosed cases which are revealed by a post-mortem examination, form, in all probability, but a comparatively small proportion of all unrecognized instances of this affection. It is probable that there is a large percentage in which the patient survives tho first and most imminent dangers of the disease, only to pass on into invalidism from chronic pelvic trouble, and finally dies, or is cured by the removal of diseased appendages, at a time when her pelvic organs have passed into a stage of inflammatory trouble (or even of chronic or acute pelvic abscess,) in which oven a 
pathologist must necessarily fail to detect any traces of tubal trouble.

In making this statement, I am well aware that I trench upon one of the uncertainties of which I spoke in my opening sentences; and I am prepared to find a strong and active difference of opinion upon the subject. I must therefore beg leave to quote the statements of more experienced men which have led me to make it.

Dr. Johnstone, of Cincinnati, in arguing this question, says of such a patient: "She is subject to all the recurrent evils of pelvic disease, and the only way to cure her is to clean them all out, the same as with any other pelvic inflammation, and before her powers are exhausted"; and reports three cases, in which he thinks that one death and two cases of serious prolonged disease were due to conservatively treated extra-nterine pregnancies. Again, he says, in speaking of the destruction of the fuetus by electricity: "Furthermore, when wo have diagnosed it correctly, and with salety to the mother destroyed both foctus and placenta, we have done little more for the future of the mother than if we had found her with a broad ligament cyst, and done nothing more than tap it. We have not only left, her to the dangers of suppuration of the present pregnancy, but to the risks of a repetition of the evil in the future."

Dr. Baldy, of Philadelphia, reports a number of cases of his own and others, in which subsequent trouble had followed an arrested extra-uterine foctation, and says: "The claim that after the death of the foctus, the trouble is past, would seem to be disproved by these cases, anounting to about eight per cent. of the whole number of women treated (cases collected), and supposed to be cases of ectopic gestation."

Thompison records a case where death of the foctus occurred between the secoud and fourth months. Later, the fotal bones were passed by the bladder.

Goelet records a case suffering from supposed pelvic hatmatocele; a vaginal incision was made upon the side of the uterus, and a foctus of about the third month, in a state of decomposition, was removed.

'I'uttle records three similar cases; Hauks, four; and the literature of the subject teems with similar instances.

In addition to these reports of cases of arrested ectopic pregnancy which are becoming frequent in literture, it seems diflicult to believe, a priori, that such masses as we have all seen removed from the abdominal cavity in cases where operation has been undertaken before rupture, can bo so completely absorbel as to leave the tube in anything but a dangerous condition. And when we renember that the diseased tubes -in which only such pregnancies occur - so constantly retain a latent power of infection for a pro. longed period of years, it seems highly probable that the contact of their secretions with the hematocele produced by an intra-ligatmentous rupture of that portion of the tube which is the seat of tubal pregnancy, must in a large proportion of cases result in the formation of a chronic pelvic abscess, even in cases in which the bones of the foctus are still capable of absorption.

It is, perhaps, as yet too soon to even estimate the frequency or infrequency of this condition; but it is certain that there is a growing disposition to assign a constantly increasing importance in the etiology of chronic pelvic disease to unrecognized and arrested tubal pregnancy.
A question of even more practical interest than the natural history of untreated cases is the possibility of obtaining an assured diaguosis of the condition. This subject can only be treated by dividing all cases into classes, in accordance with the stage to which they have advanced; that is, we must consider separately the diagnosis before rupture, immediately after rupture, and in those cases in which the accident is not recogrnized until long after rupture has occurred. With this last class belong the alleged instances of primary abdominal pregnancy.

Diagnosis before rupture. It is probable that it is the rarest of all things for the physician to see a case of ectopic gestation before any rupture has occurred. That is, the symptom which leads the patient to consult her physician is in almost all cases the pain which accompanies or immediately precedes the giving way of the walls of the tube. It must be added, however. that there is a large class of cases in which the initial rupture is incomplete, and the resulting hamorrbage is confined within a gestation sac; and it is probables that this class of cases form the bulk of those that are spoken of as having been recognized before rupture. Some such cases may often escipe recognition, from the fact that the hacmorrhage, if it occurs sulficiently early, may possible destroy the vitality of the ovum, and permit its absorption. But we have seen that there is reason to suspect that even when this result occurs it is not necessarily the most fortunate for the patient. A diagnosis which is made at this time may certainly, from a clinicul standpoint, be classilied as a diagnosis before rupture.

In another class of cases, the pathological stute is not even suspected until after the complete rupture of the walls of the tubo. 'This may occur in the initial attack of pain, or after several unrecognized attacks. It may then be so situated that the resulting hamorrhage is confined between the layers of the broad ligament, or may result in a free escape of blood into the peritoneal cavity.

In the first case, the hamorrhage may be so small that the case is classified clinically with those diagnosed before rupture, and is only distinguished as an incomplete rupture after its removal and by the patho. logist. It may be so large as to cause collapse, and the formation of an hamatocele of extreme size.

When the rupture is intra-peritoneal, it may lacerate vessels of such size as to cause promptly fatal hamorrhage; or may so far avoid the larger vessels as to cause no more hamorrhage than is capable of spontaneous arrest, and thus lead to the formation of a secoudary abdominal preguancy.

The question immediately before us now is the possibility of obtaining a diagnosis in each of these events. Diagnosis before rupture. Before any rupture has occurred, a diagnosis is very rare, because the only pationts who are likely to be seen in this stage are the very few who happen to become the victims of extrauterine pregnancy at a period when they are under troatment for some pelvic trouble. The establishment of such a diagnosis rests upon the presence of a rapidly enlarging hypersensitive tubal swelling, in connection with the rational signs of early prognancy.

After an incomplete rupture has occurred, the appear. ance of the characteristic paroxysmal colicky abdominal pains usually results in a visit to the physician, and then the question of the possibility of a diagnosis assumes a more practical aspect. 
Before entering upon the question of this possibility it may be well to again enumerate the signs upon which if made, it must rest. As in rule, the ordinary signs of pregnancy are present, namely, mammary changes, nausea, vaginal discoloration, and disturbauces of the rectal and vesical function; but, if we remember the ab estimate which is usually placed upon their value during the first three months of normal pregnancy, we shall feel that their absence is not conclusive evidence here.

An ectopic pregnancy has frequently been preceded by a period of sterility, following the birth of a child, or more often a miscarriage; but many exceptions to this rule are noted. Menstruation is apt to be profuse and irregular; and in some cases an undue frequency follows an absence of one or two periods. It may, however, be wholly regular, or entirely absent. The patient may or may not consider herself pregnant. On vaginal examination, the cervix is usually found somewhat softened, and the uterus somewhat enlarged, while in the appendages on one side or the other a tense and painful tumor is found.

Ballottement can rarely be obtained. If fragments of decidun have been found in the menstrual blood, and pronounced such by a microscopist, they would go far towards establishing a diagnosis; but this has rarely been accomplished.

$\Lambda_{t}$ tirst sight, it would seem that so extensive a collection of symptoms as this would afford conclusive evidence of the existence of ectopic pregnancy. When it is remembered how diflicult is the diaguosis of early normal pregnancy, how misleading are its sigus and symptoms, how frequently my new growth or inflammatory condition in the brond ligament is attended by sharp pain and marked heaviness, and that there is no one symptom which is absolutely characteristic of extrauterine pregnancy, it becomes evident that the problem is a complicated one.

This is further proved by an appeal to experience, and to the reported cases. Upon the one hand, we find a set of men of unquestioned authority who declare themselves able to diagnose tho presence or absence of early ectopic gestation with fuir certainty in the great majority of cases. Opposed to them are a perhaps larger number of men, whose opinion should have equal weight, who claim that such a diagnosis is impossible.

Arthur W. Johnston says, in referring to pain as the most prominent of the early symptoms: "I know that nearly every one of those colicky pains means a giving way of some part of the tube. With these pains once begun, the patient comes to seo her doctor, and then almost anybody can suspect the condition; but no one can be certain until the abdomen is opened."

Baliy states his position in three propositions: "(1) In a certain proportion of cases of extra-uterine pregnancy in the early stages, the diagnosis is easy and unmistakable. (2) In a certain (quite large) proportion of cases, sufficient symptoms are present more than to warrant a diagnosis of extra-uterine pregnancy, such a pregnancy not being present. (3) In a certain other proportion of cases, the symptoms, until rupture has occurred, are entirely wanting, or of such dubious character as in no wise to warrant such a diagnosis." He quotes cases seen by Joseph Price, P'enrose, Hirst, Coe, Johnstone, and himself, in support of his position. Gill Wylie and Vanderveer report similar cases. Thomus, with all his well-known diagnostic ability, puts himself on record upon the side of those who believe in the frequency of such mistakes in the hands of the best men. Mann says: "Tubal pregnancy has been, and therefore can be, correctly diagnosed before rupture; but this does not alter the fact that in a considerable proportion of cases, rupture takes place before the existence of pregnancy is even suspected, and that the early diagnosis before signs of tubal rupture is rare."

Kelly says: "There is yet a class of cases where we get some of the symptoms, but there remains a doubt, big or little, according as more or less of the major signs are present. Then there are other cases in which none of the symptoms are present, but the disease is found out by an accidental examination, or a sudden death." He is, however, inclined to believe that the diagnosis can usually be made.

Upon the other hand, Skene says: "Complications render diagnosis more difficult, it is true; but in an uncomplicated case of ectopic gestation, I hold that, with due care in noting the history of the case and its peculiar characteristics and the physical signs, the diagnosis can be made as positively as anything else - as pyosalpinx or ovarian cyst, for example. There may be a shadow of doubt in the minds of the best diagnosticians; but is not that so in any diagnosis?"

Wilson says, in the same discussion: "I agree with every word he (Dr. Skene) uttered, and his views are exactly my views on this subject."

Jaggard says: "The diagnosis of uncomplicated extra-uterine pregnancy is easy." And many more authorities could be quoted upon one or the other side.

I believe that the key to so extraordinary a disagreement among men equally familiar with the subject, is to be found, as is so frequently the case in such disagreements, in the fact that they are using the same words with somewhat different meanings. In studying out their various papers at length, it has seemed to une that without exception, all agree that the establishment of a diagnosis, in the sense of an ability to say, "This is ectopic preguancy, and any new growth of the ovary, or a hydro- or pyo-salpinx, can be positively excluded," is seldom easy, and frequently impossible. But that, upon the other hand, all are equally agreed that the establishment of a diagnosis, in the sense of saying, "This is probably ectopic pregnancy, and at all events is an acute enlargement in one of the uterine appendages; it is rapidly growing larger, and therefore needs immediate treatment, and this the more certainly, since it may be an ectopic pregnancy in danger of rupture," is invariably possible, whenever the symptoms have brought the case under observation sufficiently early. Such is, at all events, the result of my own experience and that of the other members of your Committee.

Diagnosis immediately after rupture, with or without the formation of an hamatocele. - When a physician of to-day is called to a caso in which the classical symptoms of concealed hamorrhinge have followed an attack of abdominal pain in a woman who may have been exposed to impregnation, the suspicion of a ruptured tubal pregnancy should be the first thing which occurs to his mind: and he should either act himself without a moment's loss of time, or with equal speed find some one else who is capable of either excluding ex tra-uterine pregnancy, or of taking tho responsibility of its treat. ment.

When the condition of collapse following paroxysmal pain is accompanied by a history of the presence of the rational and physical signs of ectopic gestation 
and an abdominal examination reveals a well-developed himatocele, there can be no question that the diagnosis is for practical purposes established ; since even if it is, as 1)r. Whitney has told us, possible that such a case may be in reality a chronic salpingitis, with hæmorrhage into the interior of the tube, the practical requirements of the case remain the same, and the differential diagnosis can only be made by the microscope.

When in the same condition even so much as a moderate enlargement of one tube, and an ill-defined bogginess in Douglas's fossa is found, the diagnosis is sufficiently well established to warrant interference.

When the history is suggestive, and the patient presents the appearance of concealed hamorrhage of serious extent, the probabilities are so much in favor of an intra-peritoneal rupture of an extra-uterine pregnancy, that the patient should not be allowed to die without an exploratory incision, unless some other adequate explanation of her condition can be found.

'The diagnosis of a pregnancy which has outlasted a rupture and become abdominal is not ordinarily an urgent matter, and is made by the recognition of foctal parts on bimanual palpation.

Treatment. - In the period before rupture. has occurred, the progress of medical science had three years ago discarded all other methods and restricted the question to the choice between electricity and colio. tomy. 'To-day, a few authorities of eminence recommend electricity as still to be considered in cases in which the diagnosis is unusually clear, when the presence of pus in the pelvis can be absolutely excluded, and when the case has certainly not advanced beyond the twelfth week. 'The great majority even of those who advocated its use in former years are, however, to-day agreed in pronouncing that it is more dangerous than coeliotomy, in view of the difficulty of eliminating mistakes in diagnosis; that it is less efficient; and that at the best it can do no more than avert the immediate dangers of the condition: to leave the woman still exposed to a risk of pelvic abscess, chronic tubal disease, and repetitions of the ectopic pregnancy.

Immediate coliotomy, so soon as the diagnosis is established, is then, in the judgment of your Committee, the only treatment which should be considered before rupture of the tube.

$\Lambda$ fter rupture, the treatment must depend upon the situation of the tear, and of the subsequent hamorrhage. If the rupture has been intra-peritoneal, so large a proportion of cases bleed to death in the first hemorrhage that there can be little doubt of the wisdom of an immediate emergency operation and the control of hamorrhage without the loss of a moment's time, by compressing the vessels of the broad ligament at the cornu of the uterus so soon as the abdomen is opened.

When the rupture is intra-ligamentous and the hamorrhage is confined between the layers of the broad ligament, much must be left to the judgment of the surgeon in the individual case. Few women actually bleed to death under these conditions; but if the tumor is, at the time when the diagnosis is made, so small that it can be readily removed, and is increasing in size, an immediate operation is probably less dangerous than the risks of further hamorrhago, in combination with those of suppuration of the tumor, or of a subsequent coeliotomy. A hurried operation, with the patient in collapse, and the relations of the organs obscured, owing to the distention of the broad ligament with a large amount of blood, may be so extremely difficult as to confuse the most expert, and it may even be advantageous to defer its performance until the recovery of the patient from the primury collapse.

If this course is chosen, the time for the subsequent interference should depend upon the progress of the case, and the rapidity with which the patient rallies. Some operators will prefer to remove the diseased appendages, with the effused blood, while it is still fresh and before there is any chance of its decomposition. The apparently more conservative course of awaiting the resorption of the hamatocele, and a secondary cocliotomy for the removal of the offending tube, if its subsequent condition renders this necessary, will commend itself to many men.

'To recapitulate, your committee believes :

(1) That the present status of medical opinion on this subject is that ectopic pregnancies are almost always, if not invariably, tubal in their origin.

(2) That the diagnosis before rupture is dependent upon the existence of a greater or less number of the following symptoms aud signs: Exposure of the patient to impregnation; a period of sterility, preceded by previous child-birth or miscarriage; the omission of one or more menstrual periods, followed by irregular over-frequent and usually profuse menstruation; the dischurge of decidual membrane, which when present is pathognomonic, but is seldom found ; the existence of intermittent, irregular, colicky pain in the abdomen ; the usual rational signs of early pregnancy in the breasts and elsewhere; slight enlargement and softening of the uterus as a whole, and the presence of a tense and sensitive tumor upon one side or behind the uterus.

(3) That a diagnosis of this condition after rupture is to be hased upon the existence of so much of the foregoing history as can be obtained, in connection with one or more attacks of severe paroxysmal, colicky pains, succeeded by sharp collapse, and the symptoms of concealed hamorrhage.

(4) 'That a diagnosis before any rupture is rare, and that the establishinent of a diagnosis before incomplete rupture occurs is beset with so many difficulties that an exact statement of the pelvic conditions, such as would be made by a pathologist upon a post-mortem examination, is usually impossible, without opening the abdomen. On the other hand, that the diagnosis of a condition which is so probably extra-uterine preguancy, and so cortainly dangerous to the patient, as to demand at least an exploratory incision, can readily be made.

(5) That the diagnosis after rupture is usually easy, and that this condition is so serious that a mere sus. picion of it should be sufficient reason for grave anxiety.

(6) That before rupture, coeliotomy is always the best treatment; electricity being less efficient and more dangerous, and ultimately less curative.

(7) That after rupture, immediate cocliotomy is indicated, except where the hamorrhage is confined within the broad ligament, and the patient is already so far collapsed that a primary operation may result fatally. When the hamorrhage is confined within the broad ligament, is still so small that the patient is in good condition, and is increasing, an immediate operation is to be recommended

Finally, that the apparent infrequency of this accident in Massachusetts, as compared with other localities, is probably due to the failure of those who have 
seen cases to give them adequate publicity, and the through the vagina or rectum, or by combined manipconsequent apathy of the general profession of this ulation.

vicinity, under whose care the great majority of such In the first group, namely, cases presenting themcases nust primarily come. selves between the first and fourth months of pregnancy, all the symptoms enumerated may be present save those given under headings 12,13 and 14 .

Group 2 may present all the symptoms enumerated, THE DIAGNOSIS OF EX'TRA-U'TERINE PREGNANCY.

BY GKORGK IIAVIR, M.b.

'THE differential diagnosis of any intra-pelvic or abdominal tumor is so uncertain and so disappointing, even when undertaken by men of large experience in abdominal work, that the statements made by certain men, notably Hanks and Kelly, regarding the ease of diagnosing cases of extra-uterine pregnancy is simply ustounding. Hanks states that a diugnosis can be made in from ninety to ninety-five per cent. of all cases; and Kelly thinks that by following certain rules, a diagnosis can be made without great difficulty.

On the other hand, Dr. Joseph Price, Dr. H. C. Coe, Dr. A. W. Johnson, Dr. 'Tuttle, Dr. 'Thomas and Dr. Gill Wylie, consider the diagnosis during the early months of extreme difficulty.

'I'he subject of diagnosis can best be considered by dividing the cases into three groups: first, cases where one is called upon to make a diagnosis during the first four mouths of pregnancy; second, cases from the fourth month to term; and, third, cuses where rupturo occurs.

'Ihe symptoms which suggest ectopic gestation can be given under fifteen headings:

(1) 'The absence of menstruation, or a flow coming on at irregular intervals, and of uncertain duration.

(2) lain of a severe and spasmodic character, which may be permanent at first, than absent for some weeks, to return later with renewed vigor.

(3) Vaginal discoloration - a symptom of some importance, yet often noticed in cases where some other form of pelvic tumor is present.

(4) General signs of pregnancy, such as nausea, enlarged and tender breasts, increase in size of the papilla, darkened aureola, milk in the breasts, ballottement, the presence of a tumor, irregular menstruation, and possibly gait.

(5) 'The history of having had a child or miscarriage. This is important, as cases occurring in nuliparous women are rure.

(6) Expulsion of decidua. This symptom is of great importance, although in the majority of cases we are not fortunate enough to have it present; or, if present, the clot and shreds of tissue are thrown away before a microscopic examination can be made.

(7) Increase in size of the uterus, with the fundus either pushed forward or to the right or left side.

(8) Elougated, soft and patulous cervix.

(9) 'The appendages on one side containing a thin walled and tender cyst. 'The fact, howover, that a tumor is felt upon both sides should have no bearing upon the diugnosis, as one of the tumors may be due to extra-uterine pregnancy, and the other to some other form of tubal, ovarian or pelvic trouble.

(10) Pulsation of vessels in neighborhood of cyst.

(11) 'The rapid increase in the size of the tumor.

(12) Presence of fotal heart sounds.

(13) Presence of placental bruit.

(14) Feeling the small parts of the child, either of pelvic inflammation or pelvic abscess. 